Rev Inv Vet Perú 2018; 29(1): 381-388

http://dx.doi.org/10.15381/rivep.v29i1.14188

\title{
Comunicación
}

\section{Peritonitis infecciosa felina: dos casos clínicos en Lima-Perú}

\author{
Feline infectious Peritonitis: two clinical cases in Lima, Peru
}

Alicia Rubio V. ${ }^{1}$, Alfonso Chavera C. ${ }^{2}$

\section{Resumen}

La Peritonitis Infecciosa Felina (PIF) es causada por un coronavirus que ocasiona una enfermedad letal en los gatos, la cual hasta el momento no tiene un tratamiento curativo. Se describen dos casos clínicos: un macho Siamés de 6 meses con apatía y anorexia y una hembra de 19 meses de pelo corto que presentó aborto y decaimiento. Ambos pacientes presentaron de leve a severa ascitis con un líquido peritoneal amarillento y proteínico compatible con PIF fase húmeda. A la necropsia se encontró ascitis y se observaron masas gelatinoides de fibrina adheridas a la serosa de hígado, bazo, pericardio, intestino y mesenterio. La histopatología mostró copioso exudado inflamatorio mixto compuesto por hilos de fibrina entremezclados con neutrófilos en diversos grados de degeneración y células plasmáticas, acompañado de severa vasculitis con hinchazón citoplasmática de las células endoteliales y efusión de líquido proteínico acidófilo.

Palabras clave: peritonitis infecciosa felina; gatos; ascitis; vasculitis

\section{AbSTRACT}

Feline Infectious Peritonitis (PIF) is a lethal disease in cats caused by a coronavirus, which until now has no curative treatment. Two clinical cases are described: a 6-month Siamese male with apathy and anorexia and a 19-month female with short hair who was presented with abortion and decay. Both patients presented mild to severe ascites with a yellowish and protein peritoneal fluid compatible with wet form of PIF. At necropsy ascites was found and fibrin gelatinoid masses adhered to the serosa of liver, spleen,

${ }^{1}$ Universidad Cientifica del Sur, Lima, Perú

${ }^{2}$ Laboratorio de Histología, Embriología y Patología Veterinaria, Facultad de Medicina Veterinaria, Universidad Nacional Mayor de San Marcos, Lima, Perú

E-mail: alicia@veterinariarubio.com.pe

Recibido: 21 de febrero de 2017

Aceptado para publicación: 26 de septiembre de 2017 
pericardium, intestine and mesentery were observed in both patients. Histopathology showed copious mixed inflammatory exudate composed of fibrin strings interspersed with neutrophils at varying degrees of degeneration and plasma cells, accompanied by severe vasculitis with cytoplasmic swelling of endothelial cells and effusion of acidophilic proteinic fluid.

Key words: feline infectious peritonitis; cats: ascites; vasculitis

\section{INTRODUCCIÓN}

La peritonitis infecciosa felina (PIF) es una enfermedad inmunomediada (Hartmann, 2010), generalmente mortal, que ataca a felinos domésticos y silvestres (Wack, 2003; Norsworthy, 2011). Es causada por el coronavirus felino (CoVF), que esta antigénicamente relacionado con el coronavirus entérico canino y el virus de la gastroenteritis transmisible de los porcinos (Kennedy y Little, 2012).

El CoVF se transmite vía fecal-oral entre los felinos, pero no presenta riesgos zoonóticos (Hartmann, 2010). Los gatos infectados eliminan el virus en las heces por un tiempo indeterminado, incluso de por vida; la mayoría permanece sano o desarrolla una leve enteritis (Addie et al., 2009). Esta enteritis es causada por el CoVF entérico que, según la respuesta inmunitaria del paciente, puede mutar y convertirse en el CoVF causante de la PIF (Chang et al., 2010; Norsworthy, 2011). Una posible inmunosupresión causada por la infección retroviral (virus de leucemia viral felina y virus de la inmunodeficiencia felina) puede reforzar la inhabilidad del paciente para controlar esta mutación (Wanderley et al., 2011). Estos cambios en las propiedades virales van acompañados de una fuerte respuesta humoral con inadecuada respuesta inmune celular; sin embargo, la producción de anticuerpos no es efectiva en la acción de eliminar el virus y esto contribuye a la enfermedad inmunomediada (Kennedy y Litttle, 2012). El porcentaje de gatos que desarrollan PIF es bajo; habiendo estudios que demuestran que ocurre en $5-15 \%$ de pacientes seropositivos (Lin et al., 2009).

Los animales afectados desarrollan dos tipos de lesiones (Hartmann, 2010): piogranulomas y vasculitis. El primero en órganos, especialmente en el sistema nervioso central, ojos y tejidos parenquimatosos, que raramente son observables por los métodos de diagnóstico por imágenes, ocasionando la llamada PIF seca; en tanto que la vasculitis genera redistribución de fluidos, ocasionando acumulación en las cavidades corporales, manifestándose como ascitis, efusión pleural, efusión pericárdica, etc, de allí que se le denomine como PIF húmeda.

Los signos clínicos varían de acuerdo con el órgano afectado y a la presencia de efusiones (Addie et al., 2009). Clínicamente el paciente presenta un deterioro progresivo, fiebre intermitente que no responde a antibióticos, inapetencia y pérdida de peso, ascitis con compromiso de los órganos abdominales y disnea en el caso de efusión pleural (Pedersen, 2009). Los hallazgos de laboratorio incluyen linfopenia y neutrofilia, pudiendo haber anemia o policitemia. Un hallazgo común es la elevación de proteínas plasmáticas debido al aumento de las gammaglobulinas, indicativo de la alta respuesta inmune causada por el virus (Addie et al., 2009; Brown et al., 2009). La relación albúmina/globulina $(\mathrm{A} / \mathrm{G})$, por lo tanto, estará disminuida en la mayoría de casos (Norsworthy, 2011), lo cual pude ayudar en el diagnóstico (Jeffery et al., 2012). Las efusiones son generalmente de color amarillento a marrón y de consistencia generalmente viscosa y mucinosa debido al alto contenido proteico (Pedersen, 2009). 
La biopsia es el único medio para confirmar PIF, por lo que muchas veces se realiza el diagnóstico durante la necropsia (Jeffery et al., 2012; Hsieh y Burney, 2014). En la histopatología se observa inflamación localizada perivascular mixta con macrófagos, neutrófilos, linfocitos y plasmocitos (Norsworthy, 2011). La presencia de anticuerpos en suero puede ser observada hasta en el 90\% en ambientes con múltiples animales (Chang et al., 2010), pero el título también puede ser fluctuante o negativo en animales enfermos (Norsworthy, 2011). Por otro lado, existen métodos de diagnóstico más especializados como PCR, inmunohistoquímica, electroforesis proteica, medición de la $\alpha-1$ ácido glicoproteína e inmunofenotipificación (Paltrinieri et al., 2004; Kipar et al., 2006; Norsworthy, 2011).

El tratamiento no es efectivo y está dirigido a controlar los signos clínicos ocasionados por la inflamación inmunomediada, para lo cual los corticosteroides son la droga de elección. Terapias con el uso de antivirales, inmunomoduladores e inmunosupresores han sido utilizados con resultados discordantes o insatisfactorios (Ishida et al., 2004; Díaz y Poma, 2009). Existe una vacuna, cuyo uso no está del todo recomendado y su efectividad no ha sido plenamente demostrada, siendo considerada una vacuna no esencial en los gatos (Richards et al., 2006; Scherk et al., 2013).

\section{Materiales y Métodos}

Se describen los casos clínicos de dos felinos domésticos que llegaron a consulta Médico Veterinaria por motivos diferentes a una clínica de referencia del distrito de Magdalena, de la ciudad de Lima, Perú en 2012.

\section{Caso 1:}

Siamés macho, entero, de seis meses, con historia de vacunaciones y antiparasitarios al día, que fuera adquirido cuatro meses antes en una tienda de mascotas. El paciente fue presentado a la consulta con anorexia parcial y letargia de 10 días. El paciente no tenía acceso a exteriores y era el único gato en la casa. El examen clínico no fue relevante y se procedió a realizar análisis de laboratorio.

\section{Caso 2:}

Gata de pelo corto de 19 meses de edad, con cerca de 65 días de gestación e historia clínica de anorexia y decaimiento por tres días. Había abortado un cachorro tres horas antes. La paciente tenía acceso a exteriores y convivía con otros cinco gatos que también tenían acceso a la calle. Al examen clínico, el abdomen se encontraba distendido, propio de una gestación, pero a la auscultación los cachorros no mostraban signos de vida. Se realizaron análisis de sangre pre-quirúrgicos, pues se programó una cesárea.

Los valores de relevancia de los hallazgos de laboratorio de ambos pacientes, se encuentran en los cuadros 1 y 2 . Al individuo del caso 1 se le realiza una ecografía abdominal, observándose leve cantidad de líquido libre, por lo que se le realizó una abdominocentesis. En la laparotomía del caso 2 se encontró abundante líquido libre abdominal con las mismas características físicas del caso 1. El líquido obtenido en ambos pacientes era amarillo oscuro con alto contenido proteico (refractometría: $6.6 \mathrm{~g} / \mathrm{dl}$ en el caso 1 y $7.0 \mathrm{~g} / \mathrm{dl}$ en el caso 2) y con propiedades de coagular (Figura 1). Microscópicamente, el líquido de ambos pacientes mostró poca celularidad, encontrándose escasos neutrófilos no degenerados.

Dada la sospecha clínica de PIF en la forma húmeda, ambos pacientes fueron sacrificados a solicitud de los propietarios. Se realizó necropsia a ambos pacientes, donde se registraron los hallazgos macroscópicos y se tomaron muestras para histopatología. 
Cuadro 1. Valores hemáticos y descarte de infección retroviral en dos gatos

\begin{tabular}{lccr}
\hline Prueba & $\begin{array}{c}\text { Caso 1: } \\
\text { Siamés }\end{array}$ & $\begin{array}{c}\text { Caso II: } \\
\text { Doméstico }\end{array}$ & \multicolumn{1}{c}{ Valores referenciales } \\
\hline Glóbulos rojos & 5.98 & 3.22 & $5.0-10.0$ millones $/ \mathrm{ml}$ \\
Hematocrito $(\mathrm{Ht})$ & 30.3 & 15 & $24-45 \%$ \\
Hemoglobina $(\mathrm{Hb})$ & 9.4 & 3.3 & $9.8-15.4 \mathrm{~g} / \mathrm{dl}$ \\
Plaquetas & 283 & 680 & $150-500 \mathrm{miles} / \mathrm{ml}$ \\
Glóbulos blancos & 13.9 & 52.67 & $5.5-19.5 \mathrm{miles} / \mathrm{ml}$ \\
Neutrófilos & 12.2 & 42.13 & $2.5-12.5 \mathrm{miles} / \mathrm{ml}$ \\
Abastonados & 0.14 & 1.58 & $0-0.3 \mathrm{miles} / \mathrm{ml}$ \\
Eosinófilos & 0 & 0.52 & $0-1.0 \mathrm{miles} / \mathrm{ml}$ \\
Basófilos & 0 & 0 & $0-0.2 \mathrm{miles} / \mathrm{ml}$ \\
Monocitos & 0.42 & 4.21 & $0-1.0 \mathrm{miles} / \mathrm{ml}$ \\
Linfocitos & 1.12 & 4.21 & $1.5-5.0 \mathrm{miles} / \mathrm{ml}$ \\
Retrovirus & Negativo & & \\
\hline
\end{tabular}

${ }^{1}$ Snap ${ }^{\circledast}$ Combo Plus FIV-FeLV (IDEXX, EEUU)

Cuadro 2. Valores de bioquímica sanguínea en dos gatos

\begin{tabular}{lccr}
\hline Prueba & $\begin{array}{c}\text { Caso 1: } \\
\text { Siamés }\end{array}$ & $\begin{array}{c}\text { Caso II: } \\
\text { Doméstico }\end{array}$ & $\begin{array}{c}\text { Valores } \\
\text { referenciales }\end{array}$ \\
\hline Creatinina & 0.82 & 0.5 & $0.5-2.0 \mathrm{mg} / \mathrm{dl}$ \\
BUN & 17 & 23 & $10-30 \mathrm{mg} / \mathrm{dl}$ \\
Urea & 38 & 49 & $18-50 \mathrm{mg} / \mathrm{dl}$ \\
Fosfatasa alcalina & 18 & 9 & $0-100 \mathrm{mg} / \mathrm{dl}$ \\
TGP & 108 & 27 & $0-60 \mathrm{U} / \mathrm{L}$ \\
GGTP & 10 & --- & $0-10 \mathrm{U} / \mathrm{L}$ \\
Proteínas totales & 8.7 & 7.1 & $5.5-7.5 \mathrm{mg} / \mathrm{dl}$ \\
Albúmina & 2.5 & 1.7 & $2.6-4.0 \mathrm{mg} / \mathrm{dl}$ \\
Globulina & 6.2 & 5.4 & $2.1-3.7 \mathrm{mg} / \mathrm{dl}$ \\
Glucosa & $(\mathrm{A} / \mathrm{G}: 0.4)$ & $(\mathrm{A} / \mathrm{G}: 0.31)$ & $60-120 \mathrm{mg} / \mathrm{dl}$ \\
\hline
\end{tabular}

A/G: Relación albúmina/globulina

\section{Resultados}

A la necropsia de ambos pacientes se encontró ascitis y masas gelatinoides de fibrina adheridas a la serosa de hígado, bazo, pericardio, intestino y mesenterio (Figura 2).
En el caso 2, las masas estaban, además, en la serosa del útero, el cual contenía cuatro fetos muertos cubiertos por líquido amniótico viscoso marrón oscuro. La presencia de este líquido corresponde en ambos casos a la llamada fase «húmeda» de PIF. 


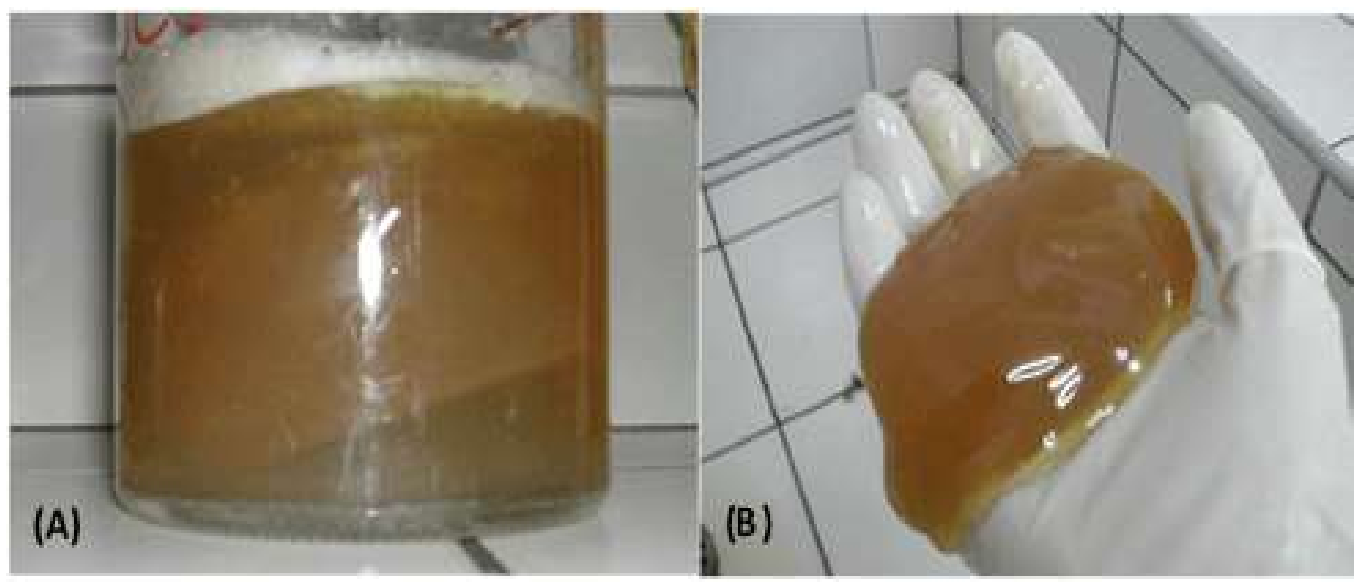

Figura 1. Líquido ascítico obtenido de ambos pacientes con sospecha de peritonitis infecciosa felina (PIF). (A) Líquido; (B) Coágulo

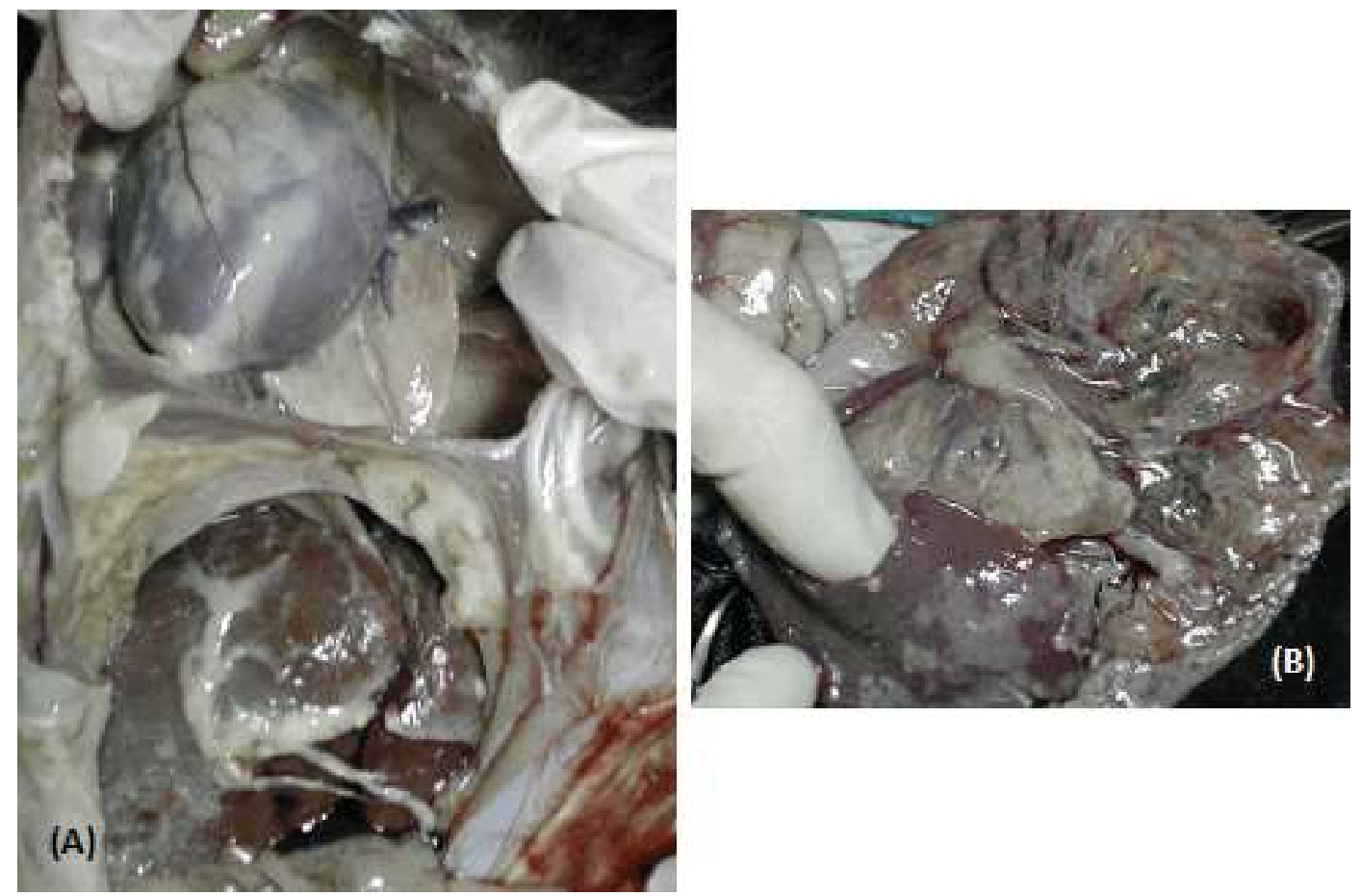

Figura 2. Hallazgos macroscópicos en dos gatos con peritonitis infecciosa felina (PIF) en su forma húmeda. (A) Fibrina adherida difusamente a la serosa cardiaca, subpericárdica y hepática. (B) Fibrina adherida difusamente en la serosa del bazo y piogranulomas en la serosa mesentérica 


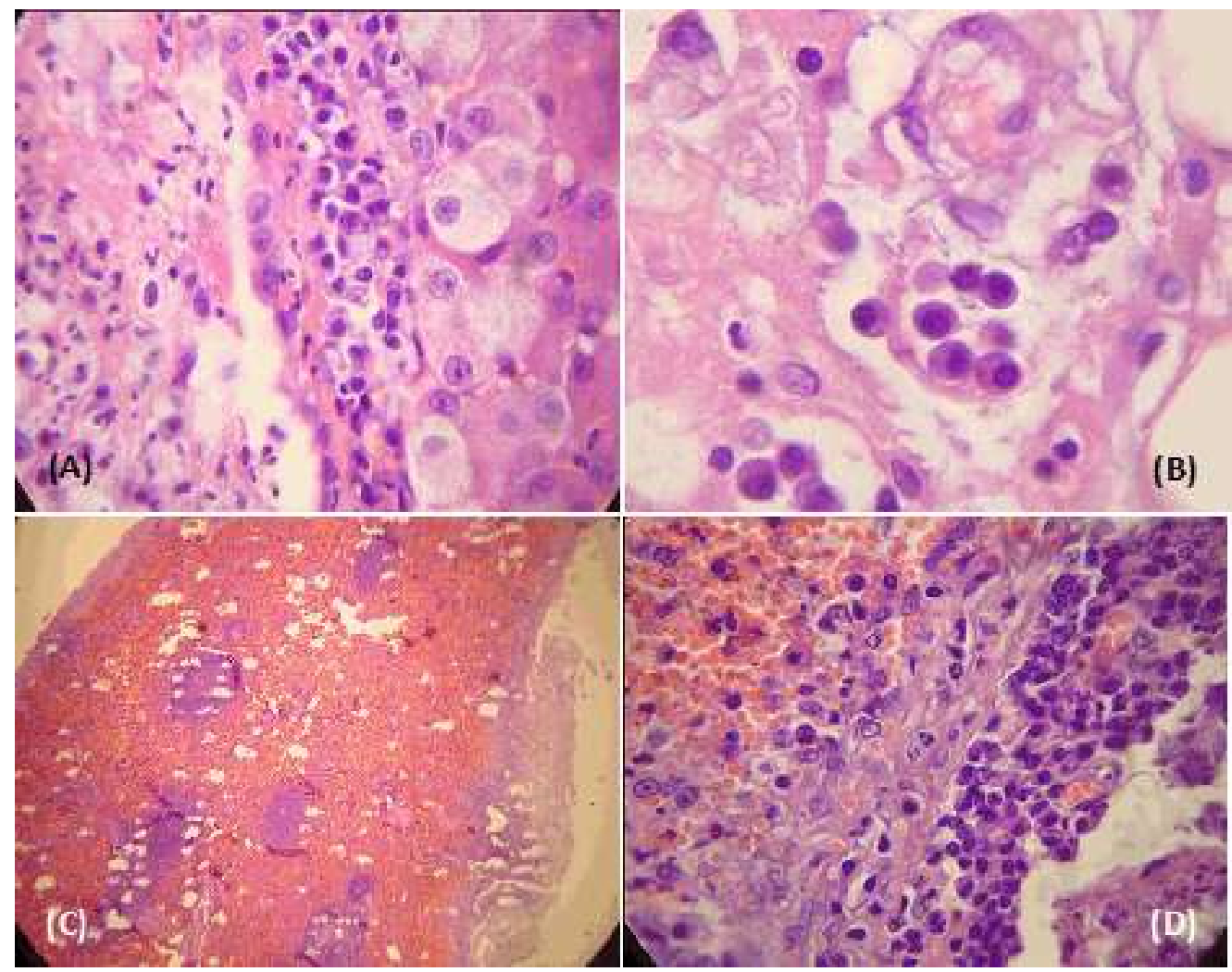

Figura 3. Hallazgos histopatológicos en dos gatos con peritonitis infecciosa felina (PIF): (A) Serosa hepática con depósito de hilos de fibrina y neutrófilos en variables diversos de degeneración; subserosa infiltrada por células plasmáticas con hepatocitos hinchados con degeneración vacuolar intracitoplasmaticas (10X, HE); (B) Hilos de fibrina rodeando a células plasmáticas y vasculitis hepática (40X, HE); (C) Exacerbación de la pulpa roja esplénica con disminución folicular linfoide, acompañándose de infiltración serosa focal (5X, HE): (D) Aproximación de la imagen C, exudado mixto compuesto por glóbulos rojos extravasados, escasos neutrófilos en diversos grados de degeneración y abundantes células plasmáticas entremezclados con hilos de fibrina depositados en serosa esplénica (10X, HE)

En las muestras histológicas se observó un copioso exudado inflamatorio mixto, compuesto por hilos de fibrina entremezclados con neutrófilos en diverso grado de degeneración y células plasmáticas, acompañándose de severa vasculitis con hinchazón citoplasmática de las células endoteliales y efusión de líquido proteínico acidófilo. Además, el hígado mostró cambio graso, zonas de necrosis y focos de exudado mononuclear y el bazo presentaba folículos linfoides en moderada depleción y linfocitolisis (Figura 3).

\section{Discusión}

El primer caso de PIF fue descrito en 1963 en Estados Unidos de América (Pedersen, 2009) y desde ese momento se ha diagnosticado en casi todo el mundo, tanto en felinos domésticos como silvestres (Paltrinieri et al., 2001; Pedersen, 2009.

El diagnóstico ante mortem de PIF suele ser un reto y se deben integrar los signos clí 
nicos y hallazgos de laboratorio (Diaz y Poma, 2009). Los dos pacientes del estudio mostraron signos clínicos inespecíficos y fue necesario recurrir a análisis complementarios para el diagnóstico final. Los resultados del hemograma fueron diferentes, pues en el caso 1 no hubo mayores cambios, a diferencia del caso 2 en donde se vio reflejado lo reportado en la literatura en referencia a la presencia de anemia y leucocitosis con neutrofilia (Pedersen, 2009).

En los resultados de bioquímica sanguínea, lo más resaltante fue la presencia de hiperglobulinemia e hipoalbuminemia en ambos casos, con una consecuente disminución del ratio albúmina/globulina. Kennedy y Little (2012) reportan que cuando este valor se acerca a 0.5 , el diagnóstico de PIF se vuelve más probable, hecho que ocurrió en ambos casos. Adicionalmente, el líquido ascítico cumplió con todas las características reportadas en la literatura (Addie et al., 2009), lo cual conllevó a la alta sospecha de PIF. Debido a que no existe un tratamiento satisfactorio probado para PIF (Norsworthy, 2011), se optó por la eutanasia, lo cual permitió realizar la necropsia de ambos con el consentimiento de los propietarios.

El hallazgo macroscópico de ascitis y masas gelatinoides de fibrina adheridas a la serosa de hígado, bazo, pericardio, intestino y mesenterio en ambos pacientes confirman la presencia de la fase húmeda que es la variante más común (Norsworthy, 2011) y, tal vez, la más fácil de diagnosticar, debido al fluido característico en cavidades corporales.

Microscópicamente, en ambos casos, se describe la presencia de un copioso exudado inflamatorio compuesto por abundante fibrina, neutrófilos en diversos grados de degeneración, gran número de plasmocitos, líquido proteínico acidófilo y vasculitis que coinciden con hallazgos reportados en la literatura (Paltrinieri et al., 2001; Diaz y Poma, 2009; Kennedy y Little, 2012).
Los signos clínicos, resultados de hemograma, las proteínas plasmáticas y las lesiones anatomo-patológicas fueron elementos suficientes para llegar al diagnóstico de Peritonitis Infecciosa Felina. Ambos casos correspondieron a la forma húmeda de la enfermedad.

\section{Literatura Citada}

1. Addie D, Belák S, Boucraut-Baralon C, Egberink H, Frymus T, GruffyddJones T, Hartmann K, et al. 2009. Feline infectious peritonitis, ABCD guidelines on prevention and management. J Feline Med Surg 11: 594604. doi: 10.1016/j.jfms.2009.05.008

2. Brown M, Troyer J, Pecon-Slattery J, Roelke M, O'Brien S. 2009. Genetics and pathogenesis of feline infectious peritonitis virus. Emerg Infect Dis 15: 1445-1452. doi: 10.3201/eid1509.081573

3. Chang H, Groot R, Egberink $H$, Rottier P. 2010. Feline infectious peritonitis: insights into feline coronavirus pathobiogenesis and epidemiology based on genetic analysis of the viral $3 \mathrm{c}$ gene. J Gen Virol 91: 415-420. doi: 10.1099/ vir.0.016485-0

4. Diaz J, Poma R. 2009. Diagnosis and clinical signs of feline infectious peritonitis in the central nervous system. Can Vet J 50: 1091-1093.

5. Ishida T, Shibanai A, Tanaka S, Uchida K, Mochizuki M. 2004. Use of recombinant feline interferon and glucocorticoid in the treatment of feline infectious peritonitis. J Feline Med Surg 6: 107-109. doi: 10.1016/j.jfms.2003.08.011

6. Hartmann K. 2010. Diagnosis and treatment of feline infectious peritonitis. In: August J (ed). Consultations in feline internal medicine. USA: Elsevier. p 62-76.

7. Hsieh B, Burney D. 2014. Feline infectious peritonitis. Clinicians brief. Febr 2014: 75-80. 
8. Jeffery U, Deitz K, Hostetter S. 2012. Positive predictive value of albumin: globulin ratio for feline infectious peritonitis in a mid-western referral hospital population. J Feline Med Surg 14: 903-905. doi: 10.1177/1098612X12454862

9. Kennedy M, Little S. 2012. Viral diseases, infectious diseases. In: Little $S$ (ed). The cat, clinical medicine and management. USA: Elsevier. p 10381047.

10. Kipar A, Baptiste K, Barth A, Reinacher M. 2006. Natural FCoV infection: cats with FIP exhibit significantly higher viral loads than healthy infected cats. J Feline Med Surg 8: 69-72. doi: 10.1016/j.jfms.2005.07.002

11. Lin C, Su B, Huang H, Lee J, Hsieh M, Chueh L. 2009. Field strain feline coronaviruses with small deletionsin ORF7b associated with both enteric infection and feline infectious peritonitis. J Feline Med Surg 11: 413-419. doi: 10.1016/j.jfms.2008.09.004

12. Norsworthy G 2011. Feline infectious peritonitis. In: Norsworthy G, Crystal M, Fooshe S, Tilley L (eds). The feline patient. $4^{\text {th }}$ ed. USA: Blackwell Publishing.p 181-183.

13. Paltrinieri S, Grieco V, Comazzi S, Cammarata Parodi M. 2001. Laboratory profiles in cats with different pathological and immunohistochemical findings due to feline infectious peritonitis
(FIP). J Feline Med Surg 3: 149-159. doi: 10.1053/jfms.2001.0126

14. Paltrinieri S, Giordano A, Ceciliani F, Sironi $G$ 2004. Tissue distribution of a feline AGP related protein (fAGPrP) in cats with feline infectious peritonitis (FIP). J Feline Med Surg 6: 99-105. doi: 10.1016/j.jfms.2003.08.012

15. Pedersen N. 2009. A review of feline infectious peritonitis virus infection: 19632008. J Feline Med Surg 11: 225-258. doi: 10.1016/j.jfms.2008.09.008

16. Richards J, Elston T, Ford R, Gaskell R, Hartmann K, Hurley, K, Lappin M, et al. 2006. The 2006 American Association of Feline Practitioners Feline Vaccines Panel report. J Am Vet Med Assoc 9: 1405-1441. doi: 10.2460/ javma.229.9.1405

17. Scherk M, Ford R, Gaskell R, Hartmann K, Hurley K, Lappin M, Levy J, et al. 2013. 2013 AAFP Feline Vaccination Advisory Panel report. J Feline Med Surg 15: 785-808. doi: 10.1177/1098612X13500429

18. Wack R. 2003. Felidae. In: Folger M, Miller E (eds). Zoo and wild animal medicine. $5^{\text {th }}$ ed. USA: Elsevier. p 491-500.

19. Wanderley L, Silva M, Peternelli F, Silva Junior A, Resende M, Almeida R. 2011. The paradox of feline coronavirus pathogenesis: a review. Advances Virol 2011: Article ID 109849. doi: 10.1155/2011/109849 